\title{
INFINITESIMAL VARIATIONS OF HODGE STRUCTURE AT INFINITY
}

\author{
JAVIER FERNANDEZ AND EDUARDO CATTANI
}

\begin{abstract}
By analyzing the local and infinitesimal behavior of degenerating polarized variations of Hodge structure the notion of infinitesimal variation of Hodge structure at infinity is introduced. It is shown that all such structures can be integrated to polarized variations of Hodge structure and that, conversely, all are limits of infinitesimal variations of Hodge structure (IVHS) at finite points. As an illustration of the rich information encoded in this new structure, some instances of the maximal dimension problem for this type of infinitesimal variation are presented and contrasted with the "classical" case of IVHS at finite points.
\end{abstract}

\section{INTRODUCTION}

It is a well known fact in mathematics that most of the interesting features of a map are encoded in its singular behavior. The Hodge theoretic version of the previous statement is that the interesting features of a polarized variation of Hodge structure (PVHS) can be described by analyzing its degenerating behavior. The purpose of this note is to start the exploration of the first order behavior of PVHS, from the perspective of a degenerating point, that is, a point at infinity.

J. Carlson, M. Green, P. Griffiths, and J. Harris introduced in [1] the idea of infinitesimal variation of Hodge structure (IVHS) as a way of associating to a PVHS an object with interesting linear algebraic invariants. Basically, if we represent locally a PVHS as an integral manifold of Griffiths' exterior differential system as in 1] or [13, then an IVHS is an integral element of the differential system.

Since IVHS are useful in the analysis of PVHS, we introduce a similar notion associated to degenerating PVHS. Infinitesimal variations of Hodge structure at infinity (IVI) are introduced in Definition 1, This idea is already implicit in the construction of compactifications in 3 as well as in 12 .

Using the local description of PVHS near infinity [14, 9, 8, we are able to prove in Theorem 3 that every IVI integrates to a PVHS and that every IVI is a limit of IVHS at infinity.

We claim that IVIs encode more refined information of a PVHS than an IVHS does for finite points. In order to illustrate this statement we look into the maximal dimension problem for IVIs, and contrast this case with the results known for IVHS.

Given the weight and Hodge numbers of a PVHS, J. Carlson, A. Kasparian and D. Toledo in [5] and R. Mayer in [13] find sharp upper bounds for the maximal dimension of an IVHS, which we call the CKTM bounds. Even though the CKTM bounds remain valid and sharp for IVIs if one considers all possible nilpotent orbits on a given period domain, considering only IVIs with underlying particular 
mixed Hodge structures or nilpotent cones leads to lower maximal dimensions, corresponding to the stronger control that the nilpotent orbit imposes on the possible IVIs.

Finally, we see that, in some cases, for a given nilpotent orbit, there are nonconjugate IVIs of maximal dimension, a phenomenon that doesn't occur for IVHS due to rigidity.

Section 2 briefly reviews some results in asymptotic Hodge theory and infinitesimal variations of Hodge structure. Section 3 introduces the notion of infinitesimal variation of Hodge structure at infinity and studies the integrability of such objects. Section 4 explores some properties of the maximal dimension problem for IVIs.

\section{Preliminaries}

The study of the degenerating behavior of variations of Hodge structure is the result of the work of P. Griffiths, P. Deligne, W. Schmid, E. Cattani and A. Kaplan, among others. We refer to 9 for a description of the subject as well as references to the original papers.

We consider a finite dimensional $\mathbb{R}$-vector space $V_{\mathbb{R}}$ and its complexification $V=\mathbb{C} \otimes V_{\mathbb{R}}$ with the induced conjugation $v \mapsto \bar{v}$. A (real) Hodge structure (HS) of weight $k$ on $V_{\mathbb{R}}$ is defined by a grading $H^{*, k-*}$ of $V$ subject to the conditions

$$
V=\oplus_{a} H^{a, k-a} \quad \text { and } \quad H^{a, k-a}=\overline{H^{k-a, a}} \text { for all } a .
$$

The numbers $h^{a, k-a}=\operatorname{dim} H^{a, k-a}$ are called the Hodge numbers of the structure. The subspaces $F^{a}=\oplus_{b \geq a} H^{b, k-b}$ form a decreasing filtration of $V$. Conversely, given such a filtration subject to $V=F^{a} \oplus \overline{F^{k-a+1}}$ for all $a$, the subspaces $H^{a, k-a}=$ $F^{a} \cap \overline{F^{k-a}}$ define a HS of weight $k$ on $V_{\mathbb{R}}$.

A polarized Hodge structure (PHS) of weight $k$ on $V_{\mathbb{R}}$ is given by a HS of weight $k$ on $V_{\mathbb{R}}, H^{*, k-*}$, and a nondegenerate bilinear form $Q$ on $V$ defined over $\mathbb{R}$, such that:

(1) $Q(u, v)=(-1)^{k} Q(v, u)$ for all $u, v \in V$,

(2) $Q\left(H^{a, k-a}, H^{b, k-b}\right)=0$ if $a+b \neq k$ and

(3) the Hermitian form $Q\left(C_{H} \cdot, \cdot\right)$ is positive definite, where $C_{H} v=i^{a-b} v$ for $v \in H^{a, b}$.

If $V$ is the vector space underlying a PHS with form $Q$, we denote by $G_{\mathbb{C}}=$ $O(V, Q)$ the group of isometries and by $G_{\mathbb{R}}$ the subgroup of $G_{\mathbb{C}}$ preserving $V_{\mathbb{R}}$. The Lie algebras of $G_{\mathbb{C}}$ and $G_{\mathbb{R}}$ are denoted by $\mathfrak{g}_{\mathbb{C}}$ and $\mathfrak{g}_{\mathbb{R}}$ respectively.

All PHS of weight $k$ and fixed Hodge numbers are parametrized by a space denoted by $\mathcal{D}$ (see [14, §3]). In order to describe $\mathcal{D}$ we consider the space of all flags of $V$ of appropriate dimension that satisfy the orthogonality condition (2). This is a subvariety of the corresponding flag manifold; it is called the compact dual space of $\mathcal{D}$ and is denoted by $\check{\mathcal{D}}$. $G_{\mathbb{C}}$ acts transitively on $\check{\mathcal{D}}$, so that $\check{\mathcal{D}} \simeq G_{\mathbb{C}} / B$ where $B \subset G_{\mathbb{C}}$ is a parabolic subgroup. The space $\mathcal{D} \subset \check{\mathcal{D}}$ corresponds to all flags that satisfy, in addition to (2), the positivity condition (3). $\mathcal{D}$ is an open subspace and $G_{\mathbb{R}}$ acts transitively on $\mathcal{D}$.

A filtration $F^{*} \in \check{\mathcal{D}}$ defines a filtration of $\mathfrak{g}_{\mathbb{C}}$ by $F^{a} \mathfrak{g}_{\mathbb{C}}=\left\{X \in \mathfrak{g}_{\mathbb{C}}: X\left(F^{b}\right) \subset\right.$ $\left.F^{a+b}\right\}$. When $F^{*} \in \mathcal{D}$, it also defines a Hodge structure of weight 0 on $\mathfrak{g}_{\mathbb{R}}$ with the grading of $\mathfrak{g}_{\mathbb{C}}$ given by

$$
\mathfrak{g}_{\mathbb{C}}^{s,-s}=\left\{X \in \mathfrak{g}_{\mathbb{C}}: X\left(H^{a, k-a}\right) \subset H^{a+s, k-a-s}\right\},
$$


where $H^{*, k-*}$ is the grading associated to $F^{*}$.

A mixed Hodge structure (MHS) on $V_{\mathbb{R}}$ consists of a pair of filtrations of $V$, $\left(W_{*}, F^{*}\right), W_{*}$ real and increasing, $F^{*}$ decreasing, such that $F^{*}$ induces a HS of weight $a$ on $\operatorname{Gr}_{a}^{W_{*}}$ for each $a$.

Given any nilpotent $N \in \mathfrak{g l}(V)$, there is a filtration $W(N)_{*}$ of $V$ called its weight filtration (see [10, page 468]). This filtration is the unique increasing filtration that satisfies $N\left(W_{l}\right) \subset W_{l-2}$ and $N^{l}: \mathrm{Gr}_{l}^{W_{*}} \rightarrow \mathrm{Gr}_{-l}^{W_{*}}$ is an isomorphism.

A polarized mixed Hodge structure (PMHS) [9, 1.16] of weight $k$ on $V_{\mathbb{R}}$ consists of a MHS $\left(W_{*}, F^{*}\right)$ on $V_{\mathbb{R}}$, a nilpotent element $N \in\left(F^{-1} \mathfrak{g}_{\mathbb{C}} \cap \mathfrak{g}_{\mathbb{R}}\right)$ and a nondegenerate bilinear form $Q$ such that

(1) $N^{k+1}=0$,

(2) $W_{*}=(W(N)[-k])_{*}$, where $W[-k]_{j}=W_{j-k}$,

(3) $Q\left(F^{a}, F^{k-a+1}\right)=0$ and,

(4) the HS of weight $k+l$ induced by $F^{*}$ on $\operatorname{ker}\left(N^{l+1}: \mathrm{Gr}_{k+l}^{W_{*}} \rightarrow \mathrm{Gr}_{k-l-2}^{W_{*}}\right)$ is polarized by $Q\left(\cdot, N^{l} \cdot\right)$.

A polarized variation of HS (PVHS) [9, Section 1] over a manifold $M$ determines a holomorphic map $\Phi: M \rightarrow \mathcal{D} / \Gamma$, where $\Gamma \subset G_{\mathbb{C}}$ is a discrete subgroup; $\Phi$ is called the period mapping. The map $\Phi$ is also locally liftable and horizontal. Horizontality in this context means that $\operatorname{Im} d \Phi$ is contained in the $G_{\mathbb{C}}$-homogeneous subbundle $T_{h} \check{\mathcal{D}} \subset T \check{\mathcal{D}}$ with fiber, over $F^{*} \in \check{\mathcal{D}}$, given by $F^{-1} \mathfrak{g}_{\mathbb{C}} / F^{0} \mathfrak{g}_{\mathbb{C}} \subset \mathfrak{g}_{\mathbb{C}} / F^{0} \mathfrak{g}_{\mathbb{C}} ; T_{h} \check{\mathcal{D}}$ is called the horizontal bundle. When $F^{*} \in \mathcal{D},\left(T_{h} \check{\mathcal{D}}\right)_{F^{*}}=\mathfrak{g}_{\mathbb{C}}^{-1,1}$ as defined by (1).

Our main interest is the asymptotic behavior of $\Phi$ near the boundary of $M$, with respect to some compactification $\bar{M}$ where $\bar{M}-M$ is a divisor with normal crossings. Such compactifications exist if, for instance, $M$ is quasiprojective. Locally at infinity we may as well replace $M$ by some product of punctured discs and discs, so that

$$
\Phi:\left(\Delta^{*}\right)^{r} \times \Delta^{m} \rightarrow \mathcal{D} / \Gamma .
$$

Since the $r$ th-power of the upper half plane, $U^{r}$ is the universal cover of $\left(\Delta^{*}\right)^{r}$, we can lift $\Phi$ to $U^{r} \times \Delta^{m}$. We still refer to this induced map by $\Phi$. We denote by $z=\left(z_{j}\right), t=\left(t_{l}\right)$ and $s=\left(s_{j}\right)$ the coordinates on $U^{r}, \Delta^{m}$ and $\left(\Delta^{*}\right)^{r}$ respectively. By definition, we have $s_{j}=e^{2 \pi i z_{j}}$.

A nilpotent orbit is a horizontal map

$$
\theta: \mathbb{C}^{r} \rightarrow \check{\mathcal{D}}, \theta(z)=\exp \left(\sum_{j=1}^{r} z_{j} N_{j}\right) \cdot F^{*}
$$

where $F^{*} \in \check{\mathcal{D}},\left\{N_{1}, \ldots, N_{r}\right\} \subset\left(F^{-1} \mathfrak{g}_{\mathbb{C}} \cap \mathfrak{g}_{\mathbb{R}}\right)$ is a commuting subset of nilpotent elements and there is $\alpha \in \mathbb{R}$ such that $\theta(z) \in \mathcal{D}$ for $\operatorname{Im}\left(z_{j}\right)>\alpha$. We usually denote a nilpotent orbit by $\left\{N_{1}, \ldots, N_{r} ; F^{*}\right\}$ and the cone $C\left(N_{1}, \ldots, N_{r}\right)=\left\{\sum \lambda_{j} N_{j}\right.$ : $\left.\lambda_{j} \in \mathbb{R}_{>0}\right\}$ is called the nilpotent cone of the orbit. Even if nilpotent orbits are analytic objects by definition, they can be algebraically characterized as asserted by the following result ([9, Theorem 2.3]) that, in turn, puts together important results of several authors.

Theorem 1. If $\left\{N_{1}, \ldots, N_{r} ; F^{*}\right\}$ is a nilpotent orbit, then

(1) $N_{j}^{k+1}=0$ where $k$ is the weight of the PHS in $\mathcal{D}$.

(2) Every $N \in C\left(N_{1}, \ldots, N_{r}\right)$ defines the same weight filtration $W_{*}^{C}$.

(3) $\left(\left(W^{C}[-k]\right)_{*}, F^{*}\right)$ is a PMHS, polarized by every $N \in C\left(N_{1}, \ldots, N_{r}\right)$. 
Conversely, if $F^{*} \in \check{\mathcal{D}}$, and $\left\{N_{1}, \ldots, N_{r}\right\}$ are commuting nilpotent elements of

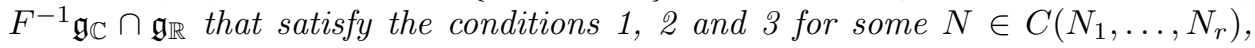
then $\left\{N_{1}, \ldots, N_{r} ; F^{*}\right\}$ is a nilpotent orbit.

By Schmid's Nilpotent Orbit Theorem [14, 4.12], there is a nilpotent orbit $\left\{N_{1}, \ldots, N_{r} ; F^{*}\right\}$ associated to any degenerating PVHS $\Phi$; in this case, $N_{j}$ is the logarithm of the unipotent part of the monodromy.

In order to make the relationship between period mappings and their nilpotent orbits more precise, recall that there is a canonical bigrading $\left\{I^{*, *}\right\}$ associated with any MHS $\left(W_{*}, F^{*}\right)$. It is uniquely characterized by the property $I^{p, q} \equiv \overline{I^{q, p}}$ $\bmod \left(\oplus_{a<p, b<q} I^{a, b}\right)$ (see [10, 2.13]). This bigrading induces, in turn, a bigrading $I^{*, *} \mathfrak{g}_{\mathbb{C}}$ of $\left(W_{*} \mathfrak{g}_{\mathbb{C}}, F^{*} \mathfrak{g}_{\mathbb{C}}\right)$.

Set

$$
\mathfrak{p}_{a}=\oplus_{q} I^{a, q} \mathfrak{g}_{\mathbb{C}} \quad \text { and } \quad \mathfrak{g}_{-}=\oplus_{a \leq-1} \mathfrak{p}_{a} .
$$

It is immediate that if $\left(W_{*}, F^{*}\right)$ is a MHS, $\left(T_{h} \check{\mathcal{D}}\right)_{F^{*}}=F^{-1} \mathfrak{g}_{\mathbb{C}} / F^{0} \mathfrak{g}_{\mathbb{C}} \simeq \mathfrak{p}_{-1}$. Also, $\mathfrak{g}_{\mathbb{C}}=\mathfrak{g}_{-} \oplus S t a b_{G_{\mathbb{C}}}\left(F^{*}\right)$, so that $\left(\mathfrak{g}_{-}, X \mapsto \exp (X) \cdot F^{*}\right)$ provides a local model for the $G_{\mathbb{C}}$-homogeneous space $\check{\mathcal{D}}$ near $F^{*}$. We recall from [9, Section 2] that we can represent a degenerating PVHS $\Phi$ by

$$
\Phi(z, t)=\exp \left(\sum_{j=1}^{r} z_{j} N_{j}\right) \cdot \exp (\Gamma(\exp (2 \pi i z), t)) \cdot F^{*}
$$

where $\left(N_{1}, \ldots, N_{r} ; F^{*}\right)$ is the nilpotent orbit and $\Gamma: \Delta^{r} \times \Delta^{m} \rightarrow \mathfrak{g}_{-}$is holomorphic. It is possible to rewrite

$$
\Phi(z, t)=\exp (X(z, t)) \cdot F^{*}
$$

for a holomorphic $X: U^{r} \times \Delta^{m} \rightarrow \mathfrak{g}_{-}$. In particular,

$$
X_{-1}(z, t)=\sum_{j=1}^{r} z_{j} N_{j}+\Gamma_{-1}(\exp (2 \pi i z), t),
$$

where the -1 subscript denotes the $\mathfrak{p}_{-1}$ part of the corresponding application.

In terms of $X$, recalling the $G_{\mathbb{C}}$-homogeneity of $T_{h} \check{\mathcal{D}}$, the horizontality of $\Phi$ is expressed by:

$$
\exp (-X) d \exp (X) \in \mathfrak{p}_{-1} \otimes T^{*}\left(U^{r} \times \Delta^{m}\right) .
$$

In fact, since the $\mathfrak{p}_{-1}$-part of $\exp (-X) d \exp (X)$ is $d X_{-1}$, the horizontality condition can be written as

$$
\exp (-X) d \exp (X)=d X_{-1} \text {. }
$$

It follows from this last expression that

$$
d X_{-1} \wedge d X_{-1}=0 .
$$

The following result, which follows from [9, Theorem 2.8] and [8, Theorem 2.7], shows that the nilpotent orbit together with the $\mathfrak{p}_{-1}$-valued holomorphic function $\Gamma_{-1}$ completely determine the local behavior of the variation:

Theorem 2. Let $\left\{N_{1}, \ldots, N_{r} ; F_{0}\right\}$ be a nilpotent orbit and $R: \Delta^{r} \times \Delta^{m} \rightarrow \mathfrak{p}_{-1}$ be a holomorphic map with $R(0,0)=0$. Define $X_{-1}(z, t)=\sum_{j=1}^{r} z_{j} N_{j}+R(s, t)$, $s_{j}=e^{2 \pi i z_{j}}$, and suppose that the differential equation (7) holds. Then, there exists a unique period mapping

$$
\Phi(s, t)=\exp \left(\frac{1}{2 \pi i} \sum_{j=1}^{r} \log \left(s_{j}\right) N_{j}\right) \cdot \exp (\Gamma(s, t)) \cdot F_{0},
$$


defined in a neighborhood of the origin in $\Delta^{r+m}$ such that $\Gamma_{-1}=R$.

The importance of this last theorem is that the information contained in a degenerating PVHS is encoded in the data of a nilpotent orbit and a holomorphic map satisfying the integrability condition (7).

Last, we turn to the first order content of a PVHS. The analysis of the differential of the period mapping at a point $F^{*} \in \mathcal{D}$ led to the following definition [1, §1.c]. An infinitesimal variation of Hodge structure (IVHS) at $F^{*} \in \mathcal{D}$ consists of a pair $(T, \delta)$, where $T$ is a finite dimensional vector space and $\delta \in \operatorname{hom}\left(T,\left(T_{h} \check{\mathcal{D}}\right)_{F^{*}}\right)=$ $\operatorname{hom}\left(T, \mathfrak{g}_{\mathbb{C}}^{-1,1}\right)$ such that $\operatorname{Im}(\delta)$ is an abelian subspace of $\mathfrak{g}_{\mathbb{C}}^{-1,1} \subset \mathfrak{g}_{\mathbb{C}}$. In other words:

$$
\begin{gathered}
\delta: T \rightarrow \oplus_{p} \operatorname{hom}\left(H^{p, k-p}, H^{p-1, k-p+1}\right) \quad \text { is linear } \\
\delta\left(\xi_{1}\right) \circ \delta\left(\xi_{2}\right)=\delta\left(\xi_{2}\right) \circ \delta\left(\xi_{1}\right) \quad \text { for all } \quad \xi_{1}, \xi_{2} \in T \\
Q(\delta(\xi) \psi, \eta)+Q(\psi, \delta(\xi) \eta)=0 \quad \text { for all } \psi \in H^{p, k-p}, \eta \in H^{p-1, k-p+1} .
\end{gathered}
$$

The 1-forms that annihilate $T_{h} \check{\mathcal{D}}$ generate the differential ideal that is known as Griffiths' exterior differential system. It turns out that, because of (9), all IVHS are integral elements of that system. It is also known [13, Proposition 3.15] that every integral element of Griffiths' system can be integrated to a germ of an integral manifold of the system which, in Hodge theoretic terms, says that all IVHS arise from (germs of) PVHS.

\section{INFINITESIMAL VARIATIONS AT INFINITY}

By analogy with the "classical" study of the first order behavior of a PVHS at a point $F^{*} \in \mathcal{D}$ via IVHS, we want to analyze the first order behavior of a degenerating PVHS at a point $F^{*} \in \check{\mathcal{D}}$, that is, at infinity.

Suppose that $\Phi$ is a degenerating PVHS with nilpotent orbit $\left\{N_{1}, \ldots, N_{r} ; F^{*}\right\}$. We will study how the tangent spaces to the image of $\Phi$ - that is, the IVHSs associated to $\Phi$ - behave as $\Phi$ degenerates at $F^{*}$.

We find the tangent spaces to the variation by computing $d \Phi_{w_{0}}$ for $w_{0}$ in a neighborhood $W$ of infinity. Following the description given in 11, pages 17 and 18] we consider:

$$
d \Phi_{w_{0}}:(T W)_{w_{0}} \rightarrow\left(T_{h} \check{\mathcal{D}}\right)_{\Phi\left(w_{0}\right)} \subset \oplus_{a} \operatorname{hom}\left(\mathrm{Gr}_{a}^{\Phi\left(w_{0}\right)}, \mathrm{Gr}_{a-1}^{\Phi\left(w_{0}\right)}\right) .
$$

If $\left\{I^{*, *}\right\}$ is the bigrading associated to the limiting MHS of $\Phi$ at $F^{*} \in \check{\mathcal{D}}$, the subspaces $J^{*}=\oplus_{q} I^{*, q} \subset V$ form a grading of $F^{*}$. Using the $G_{\mathbb{C}^{-} \text {-action on } V}$ and the form (44) for $\Phi$, we define $L^{*}=\exp \left(X\left(w_{0}\right)\right) J^{*}$, a grading of $\Phi\left(w_{0}\right)$. There

are then isomorphisms hom $\left(\operatorname{Gr}_{a}^{\Phi\left(w_{0}\right)}, \operatorname{Gr}_{a-1}^{\Phi\left(w_{0}\right)}\right) \simeq \operatorname{hom}\left(L^{a}, L^{a-1}\right) \simeq \operatorname{hom}\left(J^{a}, J^{a-1}\right)$, with the last isomorphism being conjugation by $\exp \left(X\left(w_{0}\right)\right)$. Putting together the different identifications we have

$$
d \Phi_{w_{0}}:(T W)_{w_{0}} \rightarrow \oplus_{a} \operatorname{hom}\left(J^{a}, J^{a-1}\right) \subset \mathfrak{g}_{\mathbb{C}} .
$$

We claim that under the last representation, $d \Phi=d X_{-1}$. To prove the claim, we have to show that $d \Phi_{w_{0}}=\left.\exp \left(X\left(w_{0}\right)\right) d X_{-1}\right|_{w_{0}} \exp \left(-X\left(w_{0}\right)\right)$.

Observe that if $\pi_{J^{a}}$ denotes the projection from $V$ onto the $J^{a}$ factor and, analogously, for the $L^{*}$ grading, $\pi_{L^{a}}=e^{X\left(w_{0}\right)} \pi_{J^{a}} e^{-X\left(w_{0}\right)}$.

Define $\partial_{j}=\left.\frac{\partial}{\partial w_{j}}\right|_{w_{0}}$. In order to compute $d \Phi_{w_{0}}\left(\partial_{j}\right)\left(\exp \left(X\left(w_{0}\right)\right) v_{0}\right)$ for $v_{0} \in J^{a}$, following the computation described on pages 17 and 18 of [11], a curve through 
$\exp \left(X\left(w_{0}\right)\right) v_{0}$ and contained in $\exp (X(w)) J^{a}$ for $w$ near $w_{0}$ is needed. Such a curve can be constructed considering $\exp (X(w)) v_{0}$. All together:

$$
\begin{gathered}
\exp \left(-X\left(w_{0}\right)\right) d \Phi_{w_{0}}\left(\partial_{j}\right) \exp \left(X\left(w_{0}\right)\right) v_{0}=\exp \left(-X\left(w_{0}\right)\right) \pi_{L^{a-1}}\left(\partial_{j}\left(\exp (X(w)) v_{0}\right)\right) \\
=\pi_{J^{a-1}}\left(\exp \left(-X\left(w_{0}\right)\right)\left(\partial_{j}\left(\exp (X(w)) v_{0}\right)\right)\right) \\
=\pi_{J^{a-1}}\left(\exp \left(-X\left(w_{0}\right)\right)\left(\partial_{j}(\exp (X(w))) v_{0}\right)\right) .
\end{gathered}
$$

By (66), we have $\exp \left(-X\left(w_{0}\right)\right) \partial_{j}(\exp (X(w)))=\partial_{j}\left(X_{-1}\right) \in \mathfrak{p}_{-1}$. So that

$$
\exp \left(-X\left(w_{0}\right)\right) d \Phi_{w_{0}}\left(\partial_{j}\right) \exp \left(X\left(w_{0}\right)\right) v_{0}=\left.d X_{-1}\right|_{w_{0}} v_{0}
$$

and the claim follows. Therefore, using (5),

$\operatorname{Im} d \Phi_{(s, t)}=\left.\operatorname{Im} d X_{-1}\right|_{(s, t)}=\operatorname{Span}_{\mathbb{C}}\left(N_{j}+\left.\frac{\partial \Gamma_{-1}}{\partial s_{j}}\right|_{(s, t)} 2 \pi i s_{j},\left.\frac{\partial \Gamma_{-1}}{\partial t_{l}}\right|_{(s, t)}\right.$ for all $\left.j, l\right)$.

Since $s=0$ is an accumulation point of points where $d \Phi$ has maximal rank, we can consider the limit of the corresponding tangent spaces which, by the holomorphicity of $\Gamma_{-1}$ at $(0,0)$, satisfy

$$
\lim _{s \rightarrow 0, t \rightarrow 0} \operatorname{Im} d \Phi_{(s, t)}=\operatorname{Span}_{\mathbb{C}}\left(N_{j},\left.\frac{\partial \Gamma_{-1}}{\partial t_{l}}\right|_{s=0, t=0} \text { for all } j, l\right) .
$$

If we let

$$
\mathfrak{a}_{\infty}^{\Phi}=\operatorname{Span}_{\mathbb{C}}\left(N_{j},\left.\frac{\partial \Gamma_{-1}}{\partial t_{l}}\right|_{s=0, t=0} \text { for all } j, l\right),
$$

we have just seen that the IVHSs associated to $\Phi$ - their images in the corresponding Grassmanian - have $\mathfrak{a}_{\infty}^{\Phi}$ as a limit point. Since all those subspaces are abelian, we conclude that $\mathfrak{a}_{\infty}^{\Phi}$ is also abelian.

Abstracting the features of $\mathfrak{a}_{\infty}^{\Phi}$ we arrive to the following definition.

Definition 1. Given a period domain $\mathcal{D}$, an infinitesimal variation of Hodge structure at infinity (IVI) is a pair $\left(\left\{N_{1}, \ldots, N_{r} ; F^{*}\right\}, \mathfrak{a}\right)$, where $\left\{N_{1}, \ldots, N_{r} ; F^{*}\right\}$ is a nilpotent orbit in $\check{\mathcal{D}}$ and, for $\mathfrak{p}_{-1}$ is defined by (2), $\mathfrak{a} \subset \mathfrak{p}_{-1}$ is an abelian subspace such that $\operatorname{Span}_{\mathbb{C}}\left(N_{1}, \ldots, N_{r}\right) \subset \mathfrak{a}$. The dimension of an IVI is the dimension of $\mathfrak{a}$.

Our previous discussion can be extended now to the following result relating IVIs to degenerating PVHS.

Theorem 3. Let $\Phi: U^{r} \times \Delta^{m} \rightarrow \mathcal{D}$ be a degenerating PVHS with nilpotent orbit $\left\{N_{1}, \ldots, N_{r} ; F^{*}\right\}$. Also, let $\mathfrak{a}_{\infty}^{\Phi}$ be defined by (11), where $\Gamma_{-1}$ is the holomorphic function associated to $\Phi$ by (3). Then, $\left(\left\{N_{1}, \ldots, N_{r} ; F^{*}\right\}, \mathfrak{a}_{\infty}^{\Phi}\right)$ is an IVI. Moreover, every IVI is of this form.

Proof. The previous discussion shows that $\mathfrak{a}_{\infty}^{\Phi}$ is an abelian subspace of $\mathfrak{g}_{\mathbb{C}}$ that, by construction, is contained in $\mathfrak{p}_{-1}$ and contains the nilpotent cone of the nilpotent orbit $\left\{N_{1}, \ldots, N_{r} ; F^{*}\right\}$ associated with $\Phi$. Therefore, $\left(\left\{N_{1}, \ldots, N_{r} ; F^{*}\right\}, \mathfrak{a}_{\infty}^{\Phi}\right)$ is an IVI.

Conversely, if $\left(\left\{N_{1}, \ldots, N_{r} ; F^{\cdot}\right\}, \mathfrak{a}\right)$ is an IVI, let $\left\{B_{1}, \ldots, B_{l}\right\}$ be a basis of a complement of $\operatorname{Span}_{\mathbb{C}}\left(N_{1}, \ldots, N_{r}\right)$ in $\mathfrak{a}$. Then define the map $\Gamma_{-1}: \Delta^{r} \times \mathbb{C}^{l} \rightarrow \mathfrak{p}_{-1}$ by $\Gamma_{-1}(s, t)=\sum_{j=1}^{l} t_{j} B_{j}$.

Define $X_{-1}(z, t)=\sum_{j=1}^{r} z_{j} N_{j}+\Gamma_{-1}(s, t)$, as in (5). Then, since all the elements of $\mathfrak{a}$ commute with each other, condition (7) holds so that, by Theorem 2, $X_{-1}$ extends to a degenerating PVHS $\Phi$ with the given nilpotent orbit. Since $\frac{\partial \Gamma_{-1}}{\partial t_{l}}=B_{l}$, it follows that the given IVI arises from $\Phi$. 
Remark 1. As part of the previous analysis we found that, under the corresponding identifications, $d \Phi=d X_{-1}$. In fact, under this interpretation, the integrability condition (77) says that $d X_{-1}$, and then $d \Phi$, are Higgs fields.

The notion of IVI introduced above is richer than that of IVHS in that it encodes information about the nilpotent orbit as well as the holomorphic part of a degenerating PVHS. In the next section we will illustrate this statement with several examples.

\section{Abelian subalgebras}

IVHS have appeared in connection with several geometric problems including Torelli theorems, the Noether-Lefshchetz theorems and the Yukawa coupling (in physics!). Another application has been the study of bounds on the dimension of variations of Hodge structure, as started by J. Carlson in [2. In this section we want to illustrate the notion of IVI by contrasting some examples and results with those available for IVHS.

The problem of classifying IVHS is quite hard. Still, the following result holds ([5, Theorem 1.6], [13, Theorem 4.15]).

Theorem 4. If $F^{*} \in \mathcal{D}$ and $\mathfrak{a} \subset \mathfrak{g}_{\mathbb{C}}^{-1,1} \subset \mathfrak{g}_{\mathbb{C}}$ is an abelian subspace, then $\operatorname{dim} \mathfrak{a} \leq$ $q(k, h)$, where $q$ is an explicit piecewise quadratic function of the weight $k$ and the Hodge numbers $h^{p, q}$. Furthermore, the bounds are sharp.

In the same setting, R. Mayer generalized partial results of Carlson [2] to the effect that, except for some small dimensional cases, maximal dimensional abelian subalgebras generate rigid variations [13, Theorem 5.1].

Our first observation is that IVIs satisfy the bounds of Theorem 4. Indeed, by Theorem 3, any IVI can be integrated to a PVHS of the same dimension. Moreover, we will show below that there are IVIs of the maximal dimension.

Proposition 1. For weight $k=2$ and any Hodge numbers $h^{*, k-*}$, there are IVIs whose dimension is $q(k, h)$.

Remark 2. For simplicity, we are stating Proposition 1 only for $k=2$. The result can be proved for arbitrary weight using the same techniques, as it is described in Remark 5 .

Before we start the proof of Proposition 1 we will make explicit the bounds of Theorem 4 [5, Theorem 1.6], in the case $k=2$.

(1) $h^{2,0}>1: q(2, h)=\left\{\begin{array}{l}\frac{1}{2} h^{2,0}\left(h^{1,1}-1\right)+1, \text { if } h^{1,1} \text { is odd, } \\ \frac{1}{2} h^{2,0} h^{1,1}, \text { if } h^{1,1} \text { is even. }\end{array}\right.$

(2) $h^{2,0}=1: q(2, h)=h^{1,1}$.

The following technical results are needed to prove Proposition 1.

Lemma 1. Let $V$ be a vector space underlying a PHS of weight $k$ with polarizing form $Q$ and Hodge numbers $h^{*, k-*}$. Suppose that $\left\{J^{*, *}\right\}$ is a bigrading of $V$ and $j^{a, b}=\operatorname{dim} J^{a, b}$ so that the following properties are satisfied:

(1) $J^{a, b}=\overline{J^{b, a}}$ for all $a, b$.

(2) $j^{a, b}=j^{k-b, k-a}$ for all $a, b$.

(3) $h^{p, k-p}=\sum_{b} j^{p, b}$ for all $p$.

(4) $j^{a+1, b+1} \leq j^{a, b}$ for all $a, b$ with $a+b \geq k$. 
(5) $Q\left(J^{a, b}, J^{a^{\prime}, b^{\prime}}\right)=0$ unless $a+a^{\prime}=k$ and $b+b^{\prime}=k$.

Then, if $F^{p}=\oplus_{a \geq p, b} J^{a, b}$, and $W_{l}=\oplus_{a+b \leq l} J^{a, b}$, there exist $N \in \mathfrak{g}_{\mathbb{R}}$ such that $\left(W[-k]_{*}, F^{*}, N\right)$ is a PMHS.

Proof. This is only a sketch: the details are an exercise in linear algebra. First notice that, by condition 1, $\left(W_{*}, F^{*}\right)$ defines a MHS split over $\mathbb{R}$. Then, conditions 1, 2 3 and 4 imply the existence of $N \in \mathfrak{g l}\left(V_{\mathbb{R}}\right)$ such that $W_{*}=W(N)_{*}$ and $N$ is a $(-1,-1)$ morphism of the MHS. Last, condition 5 implies that $N$ can be chosen in $\mathfrak{g}_{\mathbb{R}}$ and so that $\left(W[-k]_{*}, F^{*}, N\right)$ is a PMHS.

Lemma 2. For any weight, $k$, and Hodge numbers, $h^{*, k-*}$, let $j^{a, b} \in \mathbb{Z}_{\geq 0}$ be such that conditions 0 , 3 and 4 in Lemma 1 hold. Then there are bigradings $\left\{J^{*, *}\right\}$ of $V$ with $j^{a, b}=\operatorname{dim} J^{a, b}$ such that the rest of the conditions of Lemma 11 hold.

Remark 3. Combining Lemmas 1 and 2 we see that it suffices to set dimensions satisfying adequate compatibility conditions to ensure the existence of PMHS with bigrading of dimensions given by the given data.

Notice that by the symmetry conditions, it is sufficient to set compatible values of $j^{a, b}$ for $a+b \geq k$ and $a \geq b$. In what follows, we will usually set the values of some $j^{a, b}$, with the others determined either by symmetry or, otherwise, are 0 .

Proof of Proposition 1, We start with the case $h^{2,0}>1$.

Suppose $h^{1,1}$ is odd. There are two possibilities to consider:

- $2 h^{2,0}>h^{1,1}-1$. By Lemma 2, given dimensions $j^{2,1}=\frac{1}{2}\left(h^{1,1}-1\right), j^{2,0}=$ $h^{2,0}-\frac{1}{2}\left(h^{1,1}-1\right), j^{1,1}=1$ there are MHS $\left\{J^{*, *}\right\}$ with the right dimensions, polarized by some $N$. As an illustration of the ideas used in the proof of Lemma 2, we will construct the bigrading $\left\{J^{*, *}\right\}$ explicitly. $Q$ has signature $\left(2 h^{2,0}, h^{1,1}\right)$ so that we can split $V=V_{1} \oplus V_{2} \oplus V_{3}$ with $\operatorname{dim} V_{1}=1, \operatorname{dim} V_{2}=$ $2\left(h^{1,1}-1\right)$ and $\operatorname{dim} V_{3}=2 h^{2,0}-h^{1,1}+1$ and so that the signature of $\left.Q\right|_{V_{i}}$ is $(0,1),\left(h^{1,1}-1, h^{1,1}-1\right)$ and $\left(2 h^{2,0}-h^{1,1}+1,0\right)$ respectively. Split $V_{2}=I_{1} \oplus I_{2}$ with $I_{j}$ real and isotropic, $\operatorname{dim} I_{j}=h^{1,1}-1$. Notice that $Q$ induces $I_{2} \simeq I_{1}^{*}$. Write $I_{1}=K_{1} \oplus \overline{K_{1}}$, and under the previous isomorphism $I_{2}=K_{2} \oplus \overline{K_{2}}$ where $K_{2} \simeq K_{1}^{*}$. Finally, $V_{3}=W \oplus \bar{W}$ with $W$ isotropic, $\operatorname{dim} W=h^{2,0}-\frac{1}{2}\left(h^{1,1}-1\right)$. Now define $J^{1,1}=V_{1}, J^{2,1}=K_{1}, J^{1,2}=\overline{K_{1}}$, $J^{1,0}=\overline{K_{2}}, J^{0,1}=K_{2}, J^{2,0}=W$ and $J^{0,2}=\bar{W}$. Then the bigrading $\left\{J^{*, *}\right\}$ satisfies the conditions of Lemma 1 so that it induces a PMHS.

Any map $\tilde{X} \in \operatorname{hom}\left(J^{2,1}, J^{1,0}\right)$ can be extended to a map $X \in \mathfrak{p}_{-1}\left(\mathfrak{p}_{*}\right.$ as defined by (2) $)$ such that $\left.X\right|_{J^{1,2}} \in \operatorname{hom}\left(J^{1,2}, J^{0,1}\right)$ is dual to $\tilde{X}$, using condition [5] in Lemma 1] and $X$ vanishes elsewhere. The same argument shows that any $\tilde{\phi} \in \operatorname{hom}\left(J^{2,0}, J^{1,1}\right)$ and $\tilde{\psi} \in \operatorname{hom}\left(J^{2,0}, J^{1,0}\right)$ extend to maps $\phi, \psi \in \mathfrak{p}_{-1}$. All of this may be schematized as follows:

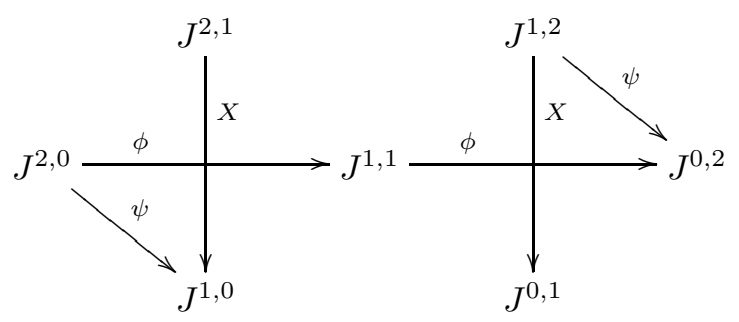


Let $\mathfrak{a}_{1}$ and $\mathfrak{a}_{2}$ be respectively the spaces of all the maps $X$ and $\psi$ constructed as above. Clearly $\mathfrak{a}_{1}$ and $\mathfrak{a}_{2}$ are abelian. Moreover, any map $\phi$ commutes with $\mathfrak{a}_{1} \oplus \mathfrak{a}_{2}$. For a fixed $\phi \neq 0$, define the abelian subspace $\mathfrak{a}=\mathfrak{a}_{1} \oplus \mathfrak{a}_{2} \oplus$ $\mathbb{C}\{\phi\} \subset \mathfrak{p}_{-1}$. We have $\operatorname{dim} \mathfrak{a}=\operatorname{dim} \mathfrak{a}_{1}+\operatorname{dim} \mathfrak{a}_{2}+1=\left(j^{2,1}\right)^{2}+j^{2,0} j^{2,1}+1=$ $h^{2,0}\left(\frac{h^{1,1}-1}{2}\right)+1$.

Any $N \in J^{-1,-1} \mathfrak{g}_{\mathbb{C}}$ automatically satisfies $N \in \mathfrak{a}_{1}$. For a given $N$ which polarizes the MHS under consideration, by Theorem 11, there are nilpotent orbits $\left\{N_{1}, \ldots, N_{r} ; F^{*}\right\}$ such that $N$ is in the relative interior of $C\left(N_{1}, \ldots, N_{r}\right)$. Since all $N_{j} \in J^{-1,-1} \mathfrak{g}_{\mathbb{C}}, N_{j} \in \mathfrak{a}_{1}$. Thus $\left(\left\{N_{1}, \ldots, N_{r} ; F^{*}\right\}, \mathfrak{a}\right)$ is an IVI.

Notice that in this case we can have a nilpotent cone of maximal dimension $\left(j^{2,1}\right)^{2}$.

- $2 h^{2,0} \leq h^{1,1}-1$. Consider a PMHS whose bigrading satisfies $j^{2,1}=h^{2,0}$, $j^{1,1}=h^{1,1}-2 h^{2,0}$. As in the previous case, any $\tilde{X} \in \operatorname{hom}\left(J^{2,1}, J^{1,0}\right)$ and $\tilde{\phi} \in \operatorname{hom}\left(J^{2,1}, J^{1,1}\right)$ extend to maps $X, \phi \in \mathfrak{p}_{-1}$. This is described by:

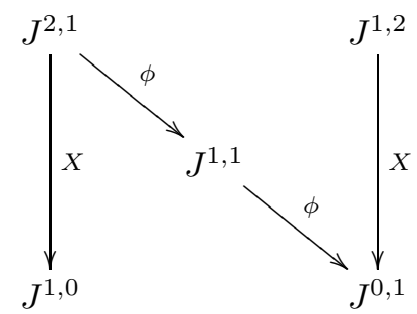

Since $\left.Q\right|_{J^{1,1}}$ is positive definite, we can choose a splitting $J^{1,1}=L \oplus K \oplus \bar{K}$ where $\operatorname{dim} L=1, K$ is isotropic and $L$ and $K$ are orthogonal.

Let $\mathfrak{a}_{1}$ and $\mathfrak{a}_{2}$ be respectively the spaces of maps $X$ and $\tau$ generated by $\tilde{X} \in \operatorname{hom}\left(J^{2,1}, J^{1,0}\right)$ and $\tilde{\tau} \in \operatorname{hom}\left(J^{2,1}, K\right)$. Then $\mathfrak{a}_{1} \oplus \mathfrak{a}_{2}$ is abelian. Also, any fixed map $\tilde{\psi} \in \operatorname{hom}\left(J^{2,1}, L\right)$ induces a map $\psi \in \mathfrak{p}_{-1}$ that commutes with $\mathfrak{a}_{1} \oplus \mathfrak{a}_{2}$. Thus $\mathfrak{a}=\mathfrak{a}_{1} \oplus \mathfrak{a}_{2} \oplus \mathbb{C}\{\psi\} \subset \mathfrak{p}_{-1}$ is an abelian subspace with $\operatorname{dim} \mathfrak{a}=\operatorname{dim} \mathfrak{a}_{1}+\operatorname{dim} \mathfrak{a}_{2}+1=\left(j^{2,1}\right)^{2}+\frac{1}{2} j^{2,1}\left(j^{1,1}-1\right)+1=\frac{1}{2} h^{2,0}\left(h^{1,1}-1\right)+1$.

The construction of the IVI then concludes as in the previous case.

The case with $h^{1,1}$ even is done along the same lines as $h^{1,1}$ odd.

Now suppose that $h^{2,0}=1$. If $h^{1,1} \geq 2$, we consider a PMHS whose bigrading has $j^{2,1}=1, j^{1,1}=h^{1,1}-2$ and is polarized by $N$ :

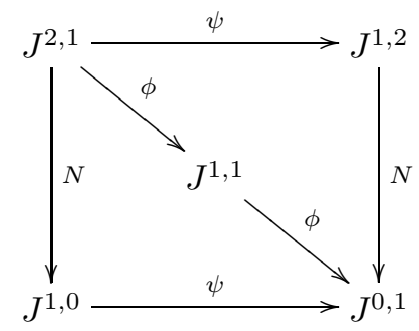

Then, there is a space of dimension $h^{1,1}-2$ of maps $\phi$, together with $\mathbb{C}\{N\}$ and $\mathbb{C}\{\psi\}$ for any fixed $\psi \neq 0$. They all commute, making an abelian space of dimension $h^{1,1}$, as required.

The cases when $h^{2,0}=1$ and $h^{1,1}<2$ are immediate but notice that there is no logarithmic part (i.e., $r=0$ ). 
Remark 4. In a few places during in the proof of Proposition 1 we picked a map among many possible choices. For instance, in the case shown in diagram (12), we chose a map $\phi$ to enlarge the abelian subspace $\mathfrak{a}_{1} \oplus \mathfrak{a}_{2}$. One may ask if it could be possible to enlarge the resulting abelian subspace even more by adding other such maps. On general grounds, the answer is no, since the dimension of the resulting abelian subspace would have to satisfy the CKTM bounds, and the examples constructed in the proof are of maximal dimension. More explicitly, in the case of diagram (12), if $\phi^{\prime}$ is like $\phi$ and commutes with $\phi$, it is easy to check that $\phi^{\prime} \in \mathbb{C}\{\phi\}$, so that further enlargement is not possible.

Remark 5. The proof of Proposition 1 in arbitrary weight follows along similar lines. Indeed, the proof of the sharpness of the bound in [5] is made by showing, for each set of Hodge numbers, a specific IVHS of the maximal dimension. This IVHS is constructed out of four types of basic examples, combined appropriately by direct sums and tensor products. Hence, it suffices to show that each one of these basic types can be realized by a IVI. In the proof of Proposition 1, we introduced two of the four basic types needed. The other two are constructed similarly and analogous results for direct sums and tensor products complete the argument.

The maximal dimension of IVHS depends, by Theorem 4 , on the weight $k$ and the Hodge numbers of the structures. Proposition 1 shows that by taking appropriate nilpotent orbits and abelian spaces, it is always possible to achieve the maximal dimension given by the CKTM bounds on a given period domain $\mathcal{D}$ with IVIs. The next example will show that, for a given period domain, the maximal dimension problem for IVIs with a given underlying MHS can be more stringent.

Example 1. Here we describe all the classes of IVIs arising as degenerations of PVHS of weight $k=2$ and $h^{2,0}=h^{1,1}=3$.

Table 1 shows the different possible cones and the maximal dimension of the IVIs in each case.

\begin{tabular}{|c|c|c|}
\hline$j^{*, *}$ & nilpotent cones & max dim of IVI \\
\hline$j^{2,0}=j^{1,1}=3$ & $\{0\}$ & 4 \\
\hline$j^{2,1}=j^{1,1}=1$ and $j^{2,0}=2$ & 1 cone of dimension 1 & 4 \\
\hline$j^{2,2}=1, j^{2,0}=2$ and $j^{1,1}=3$ & cones of dimension 1,2 and 3 & in all cases 3 \\
\hline$j^{2,2}=j^{1,1}=j^{2,1}=j^{2,0}=1$ & cones of dimension 1 and 2 & in all cases 3 \\
\hline$j^{2,2}=2, j^{2,0}=1$ and $j^{1,1}=3$ & cones of dimension 1,2 and 3 & in all cases 3 \\
\hline$j^{2,2}=j^{1,1}=3$ & cones of dimension 1,2 and 3 & in all cases 3 \\
\hline
\end{tabular}

TABLE 1. MHS, nilpotent cones and IVIs obtained when $k=2$ and $h^{2,0}=h^{1,1}=3$

Remark 6. The first two rows of Table 1 correspond to MHS where the maximal dimension of IVIs coincides with the CKTM bound. The remaining rows correspond to MHS where the maximal dimension of IVIs is smaller, for all nilpotent cones.

It is conceivable that the notion of IVI could be attached to that of MHS rather than to the nilpotent orbit as we do. The following example will show that for a given MHS, the maximal dimension of the IVIs still depends on the full nilpotent orbit. 
Example 2. Consider the MHS in weight $k=2$, defined by $j^{2,2}=j^{1,1}=2 d$. As in the previous constructions, any $\tilde{\phi} \in \operatorname{hom}\left(J^{2,2}, J^{1,1}\right)$ induces a map $\phi \in \mathfrak{p}_{-1}$. The condition for the commutativity of any two such morphisms becomes $\tilde{\phi}_{2}^{t} \tilde{\phi}_{1}=\tilde{\phi}_{1}^{t} \tilde{\phi}_{2}$, where $\tilde{\phi}^{t}$ is the dual map of $\tilde{\phi}$ under $Q$. Fix real bases of $V$ where the bilinear form $Q$ is given by $\left(\begin{array}{lll} & -\mathbb{I}_{2 d} & \mathbb{I}_{2 d} \\ \mathbb{I}_{2 d} & & \end{array}\right)$. With respect to such bases, the matrix of $\tilde{\phi}^{t}$ is the transpose of the matrix of $\tilde{\phi}$. For $1 \leq a \leq 2 d$, define $\tilde{N}_{a} \in \operatorname{hom}\left(J^{2,2}, J^{1,1}\right)$ with respect to the same bases as above, by the matrices $\tilde{N}_{a}=E_{a, a}$ whose only nonzero entry is a 1 in the $(a, a)$-position. Define also $N_{0}=\sum_{a=1}^{2 d} N_{a}$. Clearly $N_{0}$ polarizes the MHS $\left\{J^{*, *}\right\}$. Then, $\left\{N_{0} ; J^{*, *}\right\}$ and $\left\{N_{1}, \ldots, N_{2 d} ; J^{*, *}\right\}$ are nilpotent orbits whose associated MHS is $\left\{J^{*, *}\right\}$.

Now, we want to find the maximal dimension of the IVIs for these nilpotent orbits.

(1) $\left\{N_{1}, \ldots, N_{2 d} ; J^{* * *}\right\}$. In this case, the commutation with all the $N_{a}$ forces the elements of the abelian subspace containing the cone to be given by diagonal matrices in $\mathbb{C}^{2 d \times 2 d}$. So, any maximal abelian subspace has, at most, dimension $2 d$. Therefore, the maximal dimension of IVIs with the given nilpotent orbit is $2 d$.

(2) $\left\{N_{0} ; J^{*, *}\right\}$. In this case, the abelian subspaces containing the nilpotent cone are simply those containing the identity matrix. This condition forces the matrices representing $\tilde{\phi}$ to be symmetric. In particular, the subspace of all the matrices of the form

$$
\left(\begin{array}{cc}
a \mathbb{I}+i B & B \\
B & a \mathbb{I}-i B
\end{array}\right)
$$

for $B \in \mathbb{C}^{d \times d}$ symmetric and $a \in \mathbb{C}$, is abelian, contains the identity matrix and has dimension $\frac{1}{2} d(d+1)+1$. So, the maximal dimension of IVIs having the given nilpotent orbit is, at least, $\frac{1}{2} d(d+1)+1$. It will follow from Proposition 2 that this is, in fact, the maximal dimension.

Finally, since for $d \geq 3, \frac{1}{2} d(d+1)+1>2 d$, we conclude that the maximal dimension depends on the nilpotent orbit and not just on the MHS.

Remark 7. In order to study the maximal dimension of IVIs whose underlying MHS is fixed, it is enough to consider one dimensional variations, that is, nilpotent orbits with nilpotent cone generated by one element. This is so since if $\left(\left\{N_{1}, \ldots, N_{r} ; F^{*}\right\}, \mathfrak{a}\right)$ is an IVI and $N=\sum a_{j} N_{j}$ then $\left\{N ; F^{*}\right\}$ is a nilpotent orbit with the same underlying MHS, so that $\left(\left\{N ; F^{*}\right\}, \mathfrak{a}\right)$ is an IVI of the same dimension with a one dimensional nilpotent cone.

The general problem of finding the maximal dimension of IVIs with a given nilpotent orbit is quite complex. Below, we concentrate on one particular case to see some of its features. 
Consider the following MHS of weight $k$ of Hodge-Tate type polarized by $N_{0}$ :

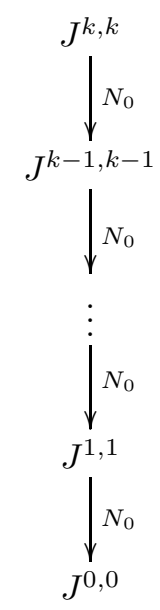

with $\operatorname{dim} J^{a, a}=n$ for $0 \leq a \leq k$. We will denote this structure by $\left\{J, N_{0}\right\}$.

First notice that any map $\phi \in \mathfrak{p}_{-1}$ commuting with the polarizing $N_{0}$ is completely determined by $\tilde{\phi}=\left.\phi\right|_{J^{k, k}}$. Indeed, since for $0 \leq a<k,\left.N_{0}\right|_{J^{a+1, a+1}}$ : $J^{a+1, a+1} \rightarrow J^{a, a}$ is an isomorphism, given $v^{a} \in J^{a, a}$ (for $0 \leq a<k$ ) there exists $v^{k} \in J^{k, k}$ such that $v^{a}=N_{0}^{k-a}\left(v^{k}\right)$. Therefore, $\phi\left(v^{a}\right)=\phi\left(N_{0}^{k-a}\left(v^{k}\right)\right)=$ $N_{0}^{k-a}\left(\phi\left(v^{k}\right)\right)$.

The problem can be phrased in terms of matrices. In order to do that, notice that $J^{k, k}$ is a pure Hodge structure of weight $2 k$ polarized by $Q_{k}(\cdot, \cdot)=Q\left(\cdot, N_{0}^{k}(\cdot)\right)$ which is symmetric nondegenerate and positive definite (on the real vector space underlying $J^{k, k}$ ). Then, there is a $Q_{k}$-orthonormal basis $\mathcal{B}_{k}=\left\{v_{1}^{k}, \ldots v_{n}^{k}\right\}$ of $J^{k, k}$. Using $N_{0}$ we define $\mathcal{B}_{a}=\left\{v_{1}^{a}, \ldots, v_{n}^{a}\right\}$, where $v_{j}^{a}=N_{0}^{k-a}\left(v_{j}^{k}\right)$ for $a=k-1, \ldots, 0$ and $j=1, \ldots, n$. The set $\mathcal{B}=\cup_{a=0}^{k} \mathcal{B}_{j}$ is a (real) basis of $\oplus_{a} J^{a, a}$. If $[\tilde{\phi}]_{\mathcal{B}_{k}, \mathcal{B}_{k-1}}$ is the matrix of $\tilde{\phi}$ with respect to the corresponding bases, the conditions $\phi \in \mathfrak{g}_{\mathbb{C}}$ and commutativity with $N_{0}$ become that $[\tilde{\phi}]_{\mathcal{B}_{k}, \mathcal{B}_{k-1}} \in \mathbb{C}^{n \times n}$ is symmetric, while commutativity of any two morphisms becomes commutativity of the respective matrices in the standard way.

In this case, the maximal dimension problem for any $k$ and $h^{a, k-a}=n$ for $0 \leq a \leq k$ reduces to that of finding maximal dimensional abelian subalgebras of symmetric matrices in $\mathfrak{g l}(n, \mathbb{C})$.

A first simplification comes from writing $\mathfrak{g l}(n, \mathbb{C})=\mathfrak{s l}(n, \mathbb{C}) \oplus \mathbb{C}$ and restricting to the $\mathfrak{s l}$ part. The bound over $\mathfrak{g l}$ will be 1 unit higher and is realized, for example, by the direct sum of a subalgebra maximizing dimension in $\mathfrak{s l}$ and the linear span of the identity matrix. Using the Cartan decomposition $\mathfrak{s l}(n, \mathbb{R})=\mathfrak{k} \oplus \mathfrak{p}$ that can be interpreted as the decomposition of trace zero matrices as the sum of antisymmetric and symmetric matrices, we reduce the maximal dimension problem for symmetric matrices to that of finding the maximal dimensional abelian subalgebras of $\mathfrak{s l}(n, \mathbb{C})$ contained in $\mathfrak{p}$. That maximal dimension has been obtained by J. Carlson and D. Toledo using root system techniques. They conclude in [7, §6] that this maximal dimension (for subalgebras in $\mathfrak{p}$ ) is

$$
\left\{\begin{array}{l}
\frac{1}{2} \alpha(\alpha+1)+\beta \text { for } n=2 \alpha+\beta>1 \text { and } \beta=0,1 \\
0 \text { for } n=1
\end{array}\right.
$$


Furthermore, they show that for even $n$ all maximal dimensional abelian subalgebras are conjugate while for odd $n$, there are two conjugacy classes. All together we have the following result.

Proposition 2. Let $\left\{N_{0} ; J\right\}$ be the nilpotent orbit of weight $k$ of (13), with $\operatorname{dim} J^{p, p}=$ $n$ for $0 \leq p \leq k$. Then, the maximal dimension of any IVI $\left(\left\{N_{0} ; J\right\}, \mathfrak{a}\right)$ is

$$
\left\{\begin{array}{l}
\frac{1}{2} \alpha(\alpha+1)+\beta+1 \text { for } n=2 \alpha+\beta>1 \text { and } \beta=0,1 . \\
1 \text { for } n=1 .
\end{array}\right.
$$

Furthermore, up to conjugation, there is only one maximal dimension IVI for $n$ even and two for $n$ odd.

Remark 8. It is easy to show either using Lie algebra theory or as a nice elementary computation [6, Theorem 5.5] that the maximal dimension of abelian subspaces of symmetric elements of $\mathfrak{g l}(n, \mathbb{R})$ is $n$. This result implies that the maximal dimension of the nilpotent cone for this MHS is $n$.

We close with a comment regarding future work. Carlson observed that the maximal dimensions given by Theorem 4 seem to be much larger than the naturally occurring PVHS: for instance, hypersurface variations are maximal PVHS of smaller dimension [4. A question remains as to what are the extra conditions that characterize the "more natural" PVHS [2]. It would be very interesting to see if the IVIs play a role in this respect since they provide a finer classification than the IVHS and so could be linked to specific degenerating behavior of the "more natural" PVHS.

\section{REFERENCES}

1. Carlson, J., Green, M., Griffiths, P., and Harris, J., Infinitesimal variations of Hodge structure. I, Compositio Math. 50 (1983), no. 2-3, 109-205.

2. Carlson, J.,Bounds on the dimension of variations of Hodge structure, Trans. Amer. Math. Soc. 294 (1986), no. 1, 45-64.

3. Carlson, J., Cattani, E., and Kaplan, A., Mixed Hodge structures and compactifications of Siegel's space (preliminary report), Journées de Géometrie Algébrique d'Angers, Juillet 1979/Algebraic Geometry, Angers, 1979, Sijthoff \& Noordhoff, Alphen aan den Rijn, 1980, pp. $77-105$.

4. Carlson, J., and Donagi, R., Hypersurface variations are maximal. I, Invent. Math. 89 (1987), no. 2, 371-374.

5. Carlson, J., Kasparian, A., and Toledo, D., Variations of Hodge structure of maximal dimension, Duke Mathematical Journal 58 (1989), no. 3, 669-694.

6. Carlson, J., and Toledo, T.,Integral manifolds, harmonic mappings, and the abelian subspace problem, Algebra-some current trends (Varna, 1986), Lecture Notes in Mathematics, no. 1352, Springer, Berlin, 1988, pp. 60-74.

7. _ Rigidity of harmonic maps of maximum rank, J. Geom. Anal. 3 (1993), no. 2, 99-140.

8. Cattani, E., and Fernandez, J.,Asymptotic Hodge theory and quantum products, Advances in algebraic geometry motivated by physics (Lowell, MA, 2000), Contemp. Math., vol. 276, Amer. Math. Soc., Providence, RI, 2001, pp. 115-136. Also, arXiv:math.AG/0011137

9. Cattani, E., and Kaplan, A. Degenerating variations of Hodge structure, Astérisque (1989), no. 179-180, 9, 67-96, Actes du Colloque de Théorie de Hodge (Luminy, 1987).

10. Cattani, E., Kaplan, A., and Schmid, W.,Degeneration of Hodge structures, Ann. of Math. (2) 123 (1986), no. 3, 457-535.

11. Griffiths, P., (ed.), Topics in transcendental algebraic geometry, Annals of Mathematics Studies, vol. 106, Princeton, NJ, Princeton University Press, 1984.

12. Kato, K, and Usui, S., Logarithmic Hodge structures and classifying spaces, The arithmetic and geometry of algebraic cycles (Banff, AB, 1998), Amer. Math. Soc., Providence, RI, 2000, pp. $115-130$. 
13. Mayer, R., Coupled contact systems and rigidity of maximal dimensional variations of Hodge structure, Trans. AMS. 352 (1999), no. 5, 2121-2144. Also, arXiv:alg-geom/9712001

14. Schmid, W., Variation of Hodge structure: the singularities of the period mapping, Invent. Math. 22 (1973), 211-319.

Instituto Balseiro, Universidad Nacional de Cuyo - C.N.E.A., Bariloche, R8402AGP, República Argentina

E-mail address: jfernand@ib.edu.ar

Department of Mathematics, University of Massachusetts, Amherst, MA 01003-9305, USA

E-mail address: cattani@math.umass.edu 